\title{
Entrevista con P. Mounoud
}

\author{
VICENTE BERMEJO \\ Universidad Complutense
}

El profesor Mounoud es uno de los investigadores más activos de la actual Sección de Psicología de la Universidad de Ginebra. Colaborador de Piaget e Inhelder durante varios años en la década de los sesenta, muy pronto «simboliza» la disidencia teórica dentro del recinto de la misma escuela piagetiana. Como él mismo apunta en la entrevista, las discrepancias surgieron principalmente de la lectura de los datos experimentales, que sugerían con claridad que el niño (y el neonato en particular) poseía mayor competencia cognitiva de la que de hecho le concedia Piaget.

Autor de numerosos trabajos científicos con proyección en Europa y Estados Unidos, su obra apenas o poco se conoce en España. De aquí la conveniencia y utilidad de esta entrevista que esperamos facilite el acceso al estudio de un autor "neopiagetiano", como él mismo se denomina en estas páginas, que rebasa las posiciones de Pascual-Leone y de Case, afirmando categóricamente no tanto el innatismo cuanto el preformismo estructural. Constructivismo y preformismo no sólo no se pondrían, sino que se complementarían.

Pregunta: Oriundo del cantón de Neuchâtel (Suiza), obtienes la licenciatura en Psicología a los veintidós años, en la Universidad de Ginebra. ¿Podrías comentar cómo o por qué elegiste los estudios de Psicologia?

Respuesta: No creo haber escapado a la regla, ya que la elección de una profesión en el ámbito social (médico/socio/psicológico) viene frecuentemente determinada por motivaciones poco claras, que manifiestan, sobre todo, un desconocimiento de sí mismo y, por supuesto, de los demás. Probablemente por esta razón tenía una viva curiosidad por intentar comprender los móviles, los determinantes del comportamiento humano y de su evolución. Durante mis estudios secundarios en el colegio, me apasioné, por ejemplo, por los Thibault, obra escrita por Roger Martin du Gard. Y estas lecturas eran ya de alguna manera psicología genética. Y además hay también, probablemente, un poco de atavismo familiar, ya que mi abuelo fue pastor protestante y mi padre médico. De todas formas, e independientemente de las razones reales que me llevaron a esta elección, nunca he lamentado la opción inicial tomada, que me ha conducido finalmente a la investigación básica y a la enseñanza.

P.: En 1962, incluso antes de terminar la Licenciatura en Psicología, eres ya profesor ayudante y colaborador de Piaget e Inbelder. ¿¿Recuerdas cómo ocurrió tu nombramiento?

Dirección del autor: Vicente Bermejo. Departamento de Psicología Evolutiva y de la Educación. Facultad de Psicologia. Universidad Complutense de Madrid. Somosaguas. 28023 Madrid. 
R.: Como es conocido, Piaget consideraba que sólo las actividades propias de la investigación podían conducir a la adquisición de una buena formación. Durante el curso 1960-1961, tuve la suerte de participar, como estudiante, en una investigación de Pierre Greco sobre la comprensión infantil de las transformaciones espaciales, e inmediatamente me «contaminé» con el virus del investigador. Posteriormente, como estudiante de tercer curso propuse un dispositivo experimental para controlar las informaciones cinemáticas en la formación de la imagen mental. Piaget e Inhelder encontraron ese dispositivo ingenioso y apreciaron igualmente el modo de realizar la investigación, y fue entonces cuando me propusieron una plaza de profesor ayudante.

P.: Durante seis años colaboras con Piaget, bien como profesor ayudante, bien como investigador en el Centre International de Epistémologie Génétique, ¿qué significó para tu formación estos años de trabajo junto al "patrón", como todos le decíamos cariñosamente en la Facultad de Psicologia y Ciencias de la Educación de Ginebra?

R.: Los años de trabajo con Piaget e Inhelder jugaron un papel capital en mi formación y en mi futuro profesional. De hecho, ha sido sólo retrospectivamente que he llegado a calibrar esa incidencia. En aquel momento las condiciones de trabajo me parecían normales $y$, sin embargo, no lo eran en absoluto, ni en la forma ni en el contenido. Las reuniones semanales de trabajo, durante las cuales el «patrón» no sólo aportaba algo sustancial, sino que además esperaba de todos una participación realmente activa, creaba un ritmo y una dinámica muy especial. Cada año se afrontaban nuevos problemas y, si bien Piaget los situaba dentro de un cuadro teórico específico, cada uno de nosotros teníamos que imaginar situaciones experimentales, realizar sondeos, encontrar técnicas de análisis, redactar informes, etc. De este modo tuve la oportunidad de realizar tres o cuatro investigaciones por año, interrogando a niños de tres a diez años según el método clínico.

$\mathrm{Y}$ en seis años he debido interrogar individualmente a más de dos mil niños. Francamente fue una formación única. Y había también la confianza y la valorización, así como las exigencias que, si bien eran normalmente flexibles, podían llegar a ser a veces muy firmes. Sólo más tarde aprendí que se trataba de características propias de un ambiente facilitador. P.: Posteriormente dejas Ginebra para realizar estudios de neuropsicología en la Universidad de Marsella. ¿Se trata de un cambio de enfoque o, simplemente, de llenar alguna laguna en tu formación ginebrina?

R.: Al finalizar mi doctorado en 1968 , pensé que había dos vías posibles para proseguir mi formación, bien en el ámbito de las matemáticas y de la lógica, siguiendo la dirección de la modelización, bien en el área de la neurofisiología para conocer mejor las bases o los fundamentos del comportamiento. Escogí entonces la segunda vía y nunca lo he lamentado. Al principio, y como ocurre siempre, con muchas ilusiones con respecto a los objetivos perseguidos. Soñé en aquel tiempo con la realización de grandes síntesis. Aquí también aprendí más tarde el papel fundamental de las ilusiones y de los sueños en los procesos genéticos.

P.: A partir de 1970 reanudas tu vida profesional en la Universidad de Ginegra, siendo profesor extraordinario de dicha Universidad en 1975, profesor ordinario en 1981 y presidente de la Sección de Psicología de la 
Facultad de Psicología y Ciencias de la Educación en 1985. Sin embargo, si mis recuerdos ginebrinos son correctos, en los años setenta "simbolizabas. la disidencia teórica dentro del recinto de la misma escuela piagetiana. ¿Cuándo, cómo y por qué se inició ese distanciamiento?

R.: Las disensiones aparecieron muy pronto y tienen varios orígenes. Sin embargo, creo que la fuente principal de mis desacuerdos y críticas radica en los datos experimentales que yo mismo he recogido, durante varios años de trabajo con Piaget. Y para mi es una buena señal que las diferencias procedan de ahí. Lo que yo descubrí es que el niño, a cualquier edad, conocía y comprendía bastante más de lo que se le quería reconocer como competencia. Y esto era tanto más verdadero cuanto que se trataba de etapas iniciales de un estadio, como el estadio denominado preoperatorio y por supuesto en el neonato. Estas constataciones me abrieron los ojos y pude detectar rápidamente en los resultados experimentales todo lo que iba en esa dirección. Finalmente, eso significó un cambio radical de perspectiva, consistente en dar mayor importancia a las competencias preformadas del neonato y al carácter généticamente programado de las grandes transformaciones de la acción y del pensamiento del niño, como del adulto y de la tercera edad. Piaget siempre tuvo miedo del espectro del preformismo, debido a que cuanto más importante fuera éste al principio, en los orígenes (de las estructuras, de los esquemas, de la organización), menos campo restaría para la construcción. Sin embargo, yo siempre he sostenido que cuanto más compleja es la organización inicial, más complejas pueden ser las influencias ambientales.

P.: ¿Qué aspectos de la teoria piagetiana han perdido, a tu juicio, aceptación o van a perderla próximamente?

R.: La genialidad de Piaget consistió en inventar la epistemología genetica, que conjuga la reflexión histórico-crítica con el estudio psicogenético. Igualmente concibió la psicogénesis de las funciones cognitivas como prolongación de adaptaciones biológicas, e introdujo la perspectiva estructuralista en la psicogénesis de los conocimientos. De este modo hizo progresar el conocimiento en estos ámbitos de una manera espectacular. Con respecto a su teoría psicológica (o de la psicogénesis), numerosos aspectos son actualmente desmentidos por los resultados empíricos, como el problema de las conservaciones, del egocentrismo, de las constancias perceptivas, etc. Numerosas cosas son cuestionadas, tanto a nivel de los datos experimentales como a nivel de las interpretaciones. Tomemos como ejemplo, en el ámbito teórico, la audaz hipótesis de Piaget de situar el origen de las operaciones lógicas en las coordinaciones generales de la acción. Yo no creo que haya actualmente muchos investigadores que sostengan esta hipótesis en el ámbito de la ontogénesis. Yo mismo entiendo que se trata de una hipótesis audaz sobre la filogénesis. Y sin embargo es una pieza maestra del edificio que continúa aún de pie. Por otra parte, su mayor aportación reside en su gran coherencia interna.

P.: ¿Podrias indicar algunas de las aportaciones piagetianas que, a tu parecer, más van a influir en psicología durante la próxima década?

R.: A medida que pasa el tiempo la influencia de Piaget es cada vez más difusa e indirecta, siendo difícil determinar lo que viene estrictamente de él o de una corriente de pensamiento. En los debates actuales sobre la concepción modular del funcionamiento cognitivo, sobre el tratamiento para- 
lelo/distribuido de la información, sobre la percepción directa, la importancia de Piaget radica en ser un polo de referencia, jen existir!

$P .:$ Supongo que actualmente existen diferentes lineas de investigación en la Sección de Psicología de Ginebra. ¿Cómo se sitúan globalmente estos grupos de trabajo con respecto a la orientación piagetiana?

R.: Después de Piaget hubo una especie de explosión de la Sección en direcciones muy divergentes y los reagrupamientos no se hicieron siempre sobre bases de afinidad científica (más bien tenían un carácter defensivo). Actualmente se han desarrollado en Ginebra varias áreas de investigación, que han encontrado su originalidad relacionándose de cerca o de lejos con Piaget y con la tradición ginebrina, en la que todos los colegas están interesados. Poco a poco emergen colaboraciones o reagrupamientos fundados sobre intereses científicos comunes. Lo que también llama la atención, y muy en la línea del espíritu piagetiano, es la dimensión interdisciplinar de todos los trabajos emprendidos. Cada profesor de la Sección tiene contactos y colaboraciones con colegas de otras disciplinas: sociólogos, lingüistas, fisiólogos, especialistas en informática, lógicos, filósofos, médicos (desde pediatras hasta especialistas en geriatría, ipasando por los psiquiatras!), etc. P.: ¿Podria hablarse en estos momentos de piagetianos «duros», de neopiagetianos $y$ de postpiagetianos?

R.: Según mis conocimientos, nadie ha sido calificado o se califica de postpiagetiano. De todas formas, se trataría, hablando con propiedad, de corrientes teóricas nuevas surgidas después de Piaget y subjetivamente independientes de él. En consecuencia, las nuevas teorías, sobre todo actualmente, no se sitúan necesariamente con respecto a las teorías pasadas. La concepción modular de Fodor (1983) podría ser un ejemplo de corriente postpiagetiana, lo mismo que el conexionismo de McClelland y Rumelhart (1986). Pero ninguno de estos autores hace referencia a Piaget, lo que, por otra parte, resulta sorprendente. De todas formas, estas concepciones no conciernen directamente al desarrollo.

En cambio, muchos psicólogos se han calificado de neopiagetianos, en concreto Jean Pascual-Leone y Robbie Case. En fin, jsería sorprendente que no hubiera conservador en el país de la conservación!

A este respecto, Piaget, en el prefacio de La Psychologie en la Encyclopédie de la Pléiade, concebía dos modos de prolongar la obra de un autor: «Esta continuación puede ser lineal, es decir que la teoría es aplicada sin modificaciones a nuevos hechos o contenidos, lo que resulta instructivo, pero no necesariamente fecundo. Normalmente, al contrario, la doctrina original se prolonga en un abanico de ramas, de modo que cada una se aleja en tal o cual punto de la trayectoria lineal, constituyéndose localmente como "anti"... Viene después una tercera etapa consistente en comparaciones o síntesis entre las ramas divergentes del abanico, de modo que se reorganiza una nueva concepción de conjunto, que constituye la más auténtica continuación de los trabajos originales propios del primero de.los tres períodos.»

P.: ¿Podría aplicarse alguno de los apelativos mencionados en la pregunta anterior a tu linea de investigación?

R.: Sin dudarlo calificaría mis trabajos y mi posición de "neopiagetianos", aunque a veces en el extranjero me siento fundamentalmente "piagetiano", 
a pesar de todas las divergencias y las críticas que yo he formulado. ¡Es un problema de escala, de amplitud de la diferencia!

P.: Los que han seguido de cerca tus publicaciones saben que desde tus primeras colaboraciones con Piaget, allá por los años sesenta, hasta boy has realizado numerosos trabajos de investigación. Al principio centras $t u$ estudio en torno al niño de cuatro a ocho años; después, en los años setenta, analizas minuciosamente algunos aspectos del desarrollo del niño pequeño; $y$ desde el inicio de esta década aproximadamente tus investigaciones, si mi lectura es correcta, a lo largo de toda la infancia, alcanzando incluso la adolescencia y el adulto. ¿Podrias señalar si, además de los problemas generales que presenta la investigación evolutiva, existen dificultades peculiares de cada edad? $Y$, en segundo lugar, ¿qué intereses más relevantes presentan cada una de estas edades?

R.: Indudablemente, existen problemas específicos en cada período evolutivo. Con respecto al periodo sensorimotor, la definición y la caracterización del «estado inicial» de las conductas del neonato constituye un problema central. Igualmente, es durante este período que se plantean con mayor agudeza los problemas vinculados a la maduración del sistema nervioso.

Durante el período de las operaciones concretas, o de las representaciones conceptuales, domina claramente el problema de la especificidad de ámbitos, al igual que el papel desempeñado por los aprendizajes escolares.

Finalmente, la adolescencia ha motivado siempre el problema de la repetición de las etapas anteriores. Pero sobre todo es en este período donde han aparecido con mayor claridad los fallos del enfoque estructural. ¿Alcanza realmente el adolescente las estructuras denominadas formales y, sobre todo, cómo evolucionan ulteriormente las capacidades cognitivas del individuo? Estos últimos años se ha clarificado cada vez más que el estudio del desarrollo, en particular el cognitivo, no puede detenerse al final de la adolescencia, de modo que el enfoque del «life span» ha obtenido una relevancia notoria.

P.: Por otra parte, en tus trabajos analizas diferentes aspectos del desarrollo: construcción del instrumento, conservaciones físicas, prensión, imagen de si mismo, levantamiento de objetos, persecución viso-manual, grafomotricidad, etc. ¿Podrias indicar los motivos cientificos que te llevaron a abordar estos grupos temáticos, y en cuál de ellos consideras más originales tus aportaciones?

R.: De una manera general, una desconfianza con respecto al lenguaje y al discurso, incluidos por supuesto los cuestionarios, y una atracción por lo que puede llamarse la acción práctica y las habilidades motoras. Pero, en realidad, la mayor parte de nuestras conductas comportan una parte importante de habilidad motora, comprendido bien entendido el lenguaje. Esta opción o estas motivaciones me condujeron a desarrollar un nuevo método, una nueva aproximación al desarrollo cognitivo. Y cuando emprendí el estudio de situaciones de resolución de problemas prácticos, tales como la construcción del instrumento en la tradición de Kohler, Guillaume y Meyerson, Rey, etc., lo hice con el objetivo de comprender mejor el desarrollo cognitivo o la inteligencia, y no solamente las habilidades motoras o sensorimotoras. Con respecto a la imagen de sí mismo, estudiada con la técnica del espejo deformante, hubo de alguna manera la misma motivación, la misma desconfianza de las técnicas fundadas en la mediación verbal. Y 
en cuanto a la aportación más original, yo creo que ha sido el método, el enfoque general más que tal o cual conducta. ¡He querido desarrollar una especie de conductismo cognitivista!

P.: ¿Podria afirmarse que existe una idea fundamental que subyace en todos tus trabajos de investigación?

R.: Una idea fundamental es ciertamente que el desarrollo cognitivo se refleja, se manifiesta también, y quizá mejor, en nuestras actividades concretas, prácticas, y no solamente en nuestros discursos, juicios y otras producciones verbales. Otra idea fundamental es que los niveles genéticos no se diferencian principalmente por su complejidad, sino por los modos de funcionamiento o estrategias, definidos por el nivel de elaboración de las representaciones. Hay que renunciar a considerar el desarrollo como una complejización creciente, o bajo el ángulo del "progreso", e insistir más en los diferentes modos de adaptación originales a unas situaciones determinadas que los sujetos de una cierta edad deben afrontar. Lo que cuenta fundamentalmente para un organismo es el poder afrontar las perturbaciones del medio ambiente. Pero la idea que más me fascina es la de la repetición de secuencias evolutivas en diferentes etapas del desarrollo, que por otra parte constituye el título de uno de mis últimos artículos.

P.: En algunas publicaciones has criticado la metodologia piagetiana empleada en las investigaciones clásicas (ej.: conservaciones). ¿En qué sentido sería deficiente esta metodología y cuál podría ser su alternativa? R.: Lo que yo he intentado hacer primeramente ha sido delimitar el método general empleado por Piaget en todas las situaciones clásicas de detección de invariantes, tanto en el ámbito lógico-matemático como en el infralógico. Este método consiste en situar el sujeto ante dos objetos o situaciones que debe considerar equivalentes. Después se transforma ante el sujeto uno de los objetos o una de las situaciones, interrogándole sobre las relaciones existentes entre la situación referencial o anterior y la situación transformada, en orden a determinar hasta qué punto domina y comprende estas transformaciones. Este método es ciertamente genial y ha revolucionado el estudio del comportamiento infantil. Sin embargo, tiene sus límites, y son estas limitaciones que he explicitado en alguno de mis trabajos. Ante todo, este método permite evidenciar la presencia de niveles de organización; pero apenas o mal permite el estudio de los mecanismos evolutivos. Esta fue la crítica principal que formulé en mi tesis doctoral de 1967 , en la que intenté rehabilitar las situaciones de la resolución de problemas como el método más adecuado para comprender los mecanismos o los procesos mismos del desarrollo. En efecto, en ciertas situaciones de resolución de problemas, el sujeto tiene la posibilidad de estimar la adecuación de sus conductas y de realizar las correcciones necesarias. Esta línea de investigación ha sido después empleada ampliamente en Ginebra, sobre todo por Inhelder.

P.: A veces se ha hablado de tu defensa de una "inteligencia práctica» en oposición a una "inteligencia conceptual $y$, sin embargo, parece existir en tus trabajos un intento insistente de relacionar acción-representación.

¿Qué bay al respecto?

R.: En el estudio del desarrollo se han opuesto dos formas de inteligencia: una, calificada de sensori-motora o práctica y, otra, de conceptual o de representativa, definiendo cada una de estas formas estadios diferentes del de- 
sarrollo. Yo me opuse violentamente a esta concepción, sosteniendo que esta oposición caracteriza las etapas de toda conducta en el desarrollo o en el aprendizaje, independientemente de la edad del sujeto. A mi juicio, hay siempre dos fases distintas en la construcción de nuevas representaciones: una, denominada «práctica» 0 "concreta» y, otra, «conceptual» o «abstracta", y esto se da tanto durante el perídodo sensori-motor como durante las etapas ulteriores. Desde mi punto de vista, acontece lo mismo con respecto a las tres formas de representación distinguidas por Jérôme Bruner; de modo que se encuentran de modo sucesivo en la elaboración de toda conducta nueva, tanto en los dos o tres primeros años de la vida como en el adulto. Por consiguiente, no hay oposición entre acción y representación. La primera es siempre mediatizada por representaciones, que pueden ser de naturaleza diferente en función de niveles distintos de elaboración. $P$.: En la teoria piagetiana juega un papel secundario el medio ambiente, al mismo tiempo que se minimiza la incidencia de lo innato en la construcción de las estructuras cognitivas. En cambio, en la concepción teórica que tú propones adquiere, a mi entender, una parte importante el preformismo y se resalta al mismo tiempo la función privilegiada del medio ambiente. ¿Cómo puede compaginarse esta «contradicción»? R.: Pienso que me he pronunciado varias veces sobre este punto. Piaget minimizó los aspectos preformados del comportamiento por temor al «preformismo", por una parte, pero sobre todo para poder explicar la emergencia de conductas que él quería considerar como nuevas desde el punto de vista estructural. El hecho de descubrir o reconocer la riqueza de las organizaciones preformadas amenaza ciertamente a teorias que intentan explicar la emergencia de conductas estructuralmente nuevas, pero no aquellas que confieren una influencia al medio ambiente. Cuanto más complejo es un organismo, más rica y diversificada es su organización, y más susceptible es, potencialmente, de ser influido por el medio.

P.: Me gustaria que dieras más detalles con respecto a la función del medio ambiente, que me parece capital en tu orientación, quizá más que el preformismo. Escribes frecuentemente que la maduración de las estructuras depende sólo de las características del medio ambiente y, a su vez, que la construcción de las organizaciones internas se realiza mediante las estructuras. Mi pregunta sería: ¿cómo incide el medio ambiente tanto en la maduración como en la construcción?

R.: Pienso que hay que distinguir un papel específico y otro no específico del medio ambiente. Actualmente se admite que la maduración del sistema nervioso no se realiza sin la presencia de ciertas condiciones mínimas de estimulación del entorno. Y éste sería el papel no específico del medio, en el sentido de que se trata más de una cantidad de estimulaciones que de la calidad de las mismas. En cambio, los caracteres específicos del medio van a ser determinantes en la construcción de representaciones. Consideramos un ejemplo: el niño pequeño inicia sus primeros pasos o emite sus primeras palabras alrededor del primer año de vida. Si no hay privaciones importantes, todos los niños manifiestan estos comportamientos en torno a los doce meses, con una variabilidad más o menos sensible determinada en parte por el papel no específico del medio. Sin embargo, la calidad de los comportamientos producidos depende de las influencias específicas del medio, de los tipos de experiencias que el niño pueda tener, de la riqueza, di- 
versidad o precisión del lenguaje de sus padres, etc. Esto es lo que va a incidir en la calidad del comportamiento infantil.

P.: Como corolario de la pregunta anterior, me gustaria saber sus implicaciones educativas; es decir, ¿qué papel juega la instrucción o enseñanza como parte, quizá privilegiada, del medio ambiente?

R.: Desde el punto de vista educativo, hay que tener en cuenta simultáneamente dos datos esenciales. Por una parte, ¿cuál es la competencia estructural del niño en función de su edad o maduración? $Y$, por otra, teniendo en cuenta estas particularidades, ¿cuáles son los métodos más adecuados para que el niño adquiera el comportamiento deseado? En esta óptica, resulta obvio que no podemos actuar del mismo modo ante un niño de cuatro años que desea aprender a leer que ante un niño de seis años. Lo que me parece fundamental es disociar lo que es función de las capacidades estructurales de las particularidades del pensamiento o del comportamiento infantil a una edad determinada, y ponerlas en relación con las particularidades de la nueva conducta que se pretende conseguir. De este modo pueden definirse requisitos tanto en sentido estricto como en sentido amplio (cf. Mounoud, 1986). Igualmente, me parece fundamental que se consideren las características individuales de la personalidad del niño en este proceso.

P.: Pasemos abora al otro polo, a la dimensión preformista de tu concepción, ¿̇existen datos válidos que la sostengan y qué ventajas tendria con respecto a la posición piagetiana, por ejemplo?

R.: Han sido los datos empíricos los que me han conducido a adoptar una posición preformista y no una preconcepción que intentaría después justificar mediante los datos experimentales. Todas las investigaciones realizadas sobre el neonato durante los veinte últimos años muestran la complejidad de sus conductas y su carácter preadaptado con respecto al medio, incluso cuando el niño es fundamentalmente un individuo dependiente. Pero el problema no es tanto lo que es preformado cuando el origen de esta organización y su potencial evolutivo. Piaget ha reconocido siempre la existencia de estructuras preformadas y ha admitido incluso el haber subestimado su importancia. Con respecto a la consideración de estructuras nuevas, Piaget las describe al principio del periodo sensori-motor como resultantes de la coordinación de esquemas mediante asimilación recíproca, y esta estructura de las coordinaciones las considera como una emergencia procedente de la interacción entre los esquemas reflejos iniciales, transformados estos mismos por la interacción con el medio. Por consiguiente, la estructura de estas coordinaciones, que Piaget consideraba «nuevas», existe desde el nacimiento, de modo que lo que construye el bebé es, pues, otra cosa.

P.: Cuando afirmas que tanto los códigos cognitivos como los instrumentos cognitivos para codificar son innatos y no adquiridos, ¿qué apoyatura teórico-empirica tiene esta afirmación?

R.: Los receptores sensoriales han sido descritos desde principios de siglo como instrumentos de codificación de energías específicas, a las que son sensibles (reaccionan). Además, se sabe que el sistema nervioso efectúa en diferentes niveles (diferentes centros) codificaciones sucesivas de estas informaciones recibidas. Ahora bien, la estructura de las codificaciones realizadas por estos centros es preformada; en cambio, los contenidos o los 
aspectos de la realidad codificados dependen, por supuesto, del entorno en que vive el individuo, y de la naturaleza y calidad de las experiencias personales.

P.: Las actividades de organización y de sintesis son efectuadas por estructuras preformadas $y$, sin embargo, esas actividades se diferencian considerablemente según sean realizadas por el niño pequeño o por el adolescente. ¿Cómo es posible que las mismas estructuras produzcan funciones $o$ actividades tan diversas de manera sistemática? $Y$ si las actividades de organización en el adolescente, por ejemplo, son llevadas a cabo por organizaciones anteriores no preformada, entonces, ¿̇ué interés tendria hablar de estructuras preformadas más allá de los primeros comportamientos del niño pequeño?

R.: La capacidad de hacer síntesis, o de integrar, o de coordinar informaciones parciales es una capacidad estructural de nuestro organismo. Pero las sintesis realizadas son función de los contenidos a los que se enfrenta el individuo. Consideremos un ejemplo: las producciones de las primeras palabras del niño resultan claramente de la síntesis de numerosos elementos del discurso (segmentos y suprasegmentos), elaborados anteriormente durante los doce primeros meses de la vida. Si el organismo funciona correctamente y el medio posee las características satisfactorias, el niño producirá sus primeras palabras; pero la calidad de estas síntesis dependerá de la calidad de los productos (discurso) del entorno del niño. Si los padres alteran las palabras, es obvio que estas capacidades infantiles no van a corregir las mencionadas defectuosidades.

P.: Se habla a veces, y desde distintos puntos de vista, de la relación entre capacidad innata y el dominio o utilización de esa capacidad, que quizá sea distinto del binomio conocimiento implícito-explícito propuesto por otros autores (Gelman, por ejemplo). ¿Podría determinarse empíricamente esta distinción?

R.: Con respecto a las competencias estructurales del niño es obvio que no se manifiestan, si no son solicitadas. Si consideramos que las competencias para realizar la segmentación fonemática de las palabras no son accesibles al niño antes de los seis años en el plano de la representación conceptual, esto no quiere decir que vaya a manifestar espontáneamente estas competencias, sin la confrontación con situaciones que exijan tales competencias. Si no se enseña a leer al niño de seis años, no actualizará sus competencias y seguirá siendo un analfabeto. Por tanto, las capacidades estructurales definen las posibilidades, pero son las experiencias las que actualizan o no estas posibilidades.

P.: Una de tus aportaciones más interesantes y atractiva es, a mi parecer, tu concepción de la representación, que actualmente es uno de los temas centrales de la Psicología cognitiva. ¿Podrias indicar con cierto detalle el proceso de construcción de la representación y si los distintos tipos de la misma se excluyen o pueden coexistir a lo largo de la vida?

R.: El niño, dotado de capacidades nuevas de codificación, no puede súbitamente "transcribir" o "transponer» o "traducir» sus representaciones anteriores. Al contrario, se trata de un proceso largo que hemos descrito en dos etapas o fases principales.

En un primer momento (primera revolución), el niño elabora lo que hemos llamado representaciones elementales o parciales de la realidad que le 
rodea. Estas representaciones son de naturaleza más bien analógica y, en función de transformaciones internas, se coordinan o se integran después para constituir representaciones globales o totales, rígidas y no descomponibles, que permiten la identificación de objetos, de personas y de situaciones de una manera singular.

En un segundo momento (segunda revolución), estas representaciones se descomponen én elementos más abstractos, que hacen posible la relación entre las partes de un objeto o entre varios objetos con respecto a algunas de sus dimensiones. $Y$ en este caso, las representaciones son más abstractas. $P .:$ Si he entendido bien, las representaciones sensoriales serian innatas o preformadas, mientras que las restantes (perceptivas, conceptuales y formales) serian construidas. ¿̇ qué se debe esta diferencia genética, $y$ realmente se trata en todos los casos de fenómenos cognitivos homólogos?

R.: Esta distinción es totalmente relativa. De un modo general, las representaciones internas de un organismo resultan necesariamente de una construcción. Ahora bien, ésta puede ser resultado de la filogénesis, de la embriogénesis o de la ontogénesis. Es evidente que el neonato llega al mundo con representaciones preformadas, es decir, desde el nacimiento ciertas configuraciones de indicios generan comportamientos específicos, de modo que todo ocurre como si estas configuraciones tuvieran desde el primer momento una significación para el niño. Así, por ejemplo, en las coordinaciones viso-auditivas parece como si el niño girase la cabeza en la dirección de la estimulación auditiva para ver el origen de esta estimulación; o bien en las imitaciones precoces, que ocurre como si el bebé fuera capaz de establecer una correspondencia entre las partes de su cara y los elementos del espectáculo que tiene delante. Y sin embargo, esta homologación de las partes de su cara con las de la cara del otro es una conducta cuya aparición se suponía posterior al año del niño. Nuestra interpretación es que la conducta del neonato y la del niño de 1 año deben interpretarse como mediatizadas por representaciones diferentes. Las primeras, que hemos llamado sensoriales, resultarían de una construcción que se realiza en el curso de la filogénesis y/o de la embriogénesis; mientras que la segundas, denominadas perceptias, resultarían de la ontogénesis y, por supuesto, se apoyarian en las anteriores. Y para mayor precisión, me parece mejor calificar las representaciones sensoriales de preformadas, en vez de innatas, a pesar de lo que he escrito en otras ocasiones.

P.: Insistes frecuentemente, como de una diferencia que te distancia de la teoria piagetiana, en la vinculación existente entre estructuras y contenidos que, por otra parte, resultaria obvia en el caso de las estructuras reflejas; pero es menos evidente con respecto a estructuras posteriores desde el punto de vista piagetiano. ¿Dónde residiría la conveniencia o necesidad de esta vinculación?

R.: Piaget denomina estructuras en psicología principalmente a dos realidades distintas. De una parte, las operaciones lógico-matemáticas y, de otra, las coordinaciones generales de la acción, siendo las primeras fuente de las segundas (cf. abstracción reflexionante). Desde mi punto de vista, tanto las coordinaciones de acción como las operaciones lógicas, son preformadas y están vinculadas a contenidos particulares. Sin embargo, estos contenidos pueden ser más o menos específicos o más o menos generales. Y cuanto 
más generales son los contenidos a los que se aplican las acciones o las operaciones, más se tiene la impresión de que las coordinaciones o las operaciones son independientes de los contenidos.

P.: Enlazando con lo dicho anteriormente, se ha vertido mucha tinta en torno a los famosos desfases borizontales, que boy están encontrando, a mi juicio, la explicación adecuada en psicología cognitiva. ¿Qué opinas de estos desfases?

R.: Generalmente se considera el concepto de desfase horizontal como algo no ambiguo. En el cuadro piagetiano se define con bastante precisión como la aplicación de una misma estructura a contenidos diferentes. Pero no se hace distinción alguna con respecto a las diferentes categorías de contenidos, a los diferentes tipos de afrontar esos contenidos, a los diferentes problemas que pueden plantearse en relación a esos contenidos. Piaget se interesó en la génesis de los instrumentos generales del conocimiento, pero no en el aprendizaje de tales a cuales conocimientos o saberes particulares. Así consideró siempre que estos instrumentos del conocimiento o estructuras lógicas eran en gran parte independientes de los saberes o aprendizajes escolares. Sin embargo, no se puede adoptar hoy este punto de vista, siendo imprescindible la introducción de ciertas distinciones. Para Piaget, el niño, en su interacción con el medio ambiente, puede dominar ciertas dimensiones o categorías antes que otras: las dimensiones numéricas antes que las espaciales, por ejemplo, a pesar del isomorfismo de las estructuras subyacentes (postuladas por Piaget) e independientemente del hecho de haber estado en contacto más o menos con estas realidades. En este caso, Piaget explica el desfase por la diferencia general que existiria entre las dimensiones lógico-matemáticas "discretas» y las dimensiones espacio-temporales «continuas». De modo que el continuo físico supondría una discretización (o segmentación), mientras que no sería el caso en el ámbito lógicomatemático, que por definición concierne a objetos discretos, es decir, es un ámbito discreto por naturaleza, lo que explicaría el desfase. Ahora bien, esta distinción es sorprendente en Piaget. Desde mi punto de vista, la realidad en su conjunto es objeto de discretización o segmentación. Pero más allá de esta distinción existen miles de maneras de interrogar al niño sobre el número y la longitud.

Tomemos otro ejemplo de desfase horizontal, referente a la capacidad para dominar la inclusión. Piaget ha estudiado esta capacidad mediante diferentes materiales: perlas, animales, flores, etc. En este caso, Piaget hipotetiza que los desfases se deben a la familiaridad de los niños con estos ámbitos (o contenidos) y que la capacidad para dominar la inclusión depende de los conocimientos prealables en torno a las especies animales o vegetales, etc. Y el error está, desde mi punto de vista, en haber hecho de estos desfases una peculiaridad del estadio de las operaciones concretas.

Las concepciones dominantes actualmente en Psicología evolutiva consideran que los conocimientos pertenecientes a diferentes ámbitos se desarrollan más o menos independientemente los unos de los otros (dominio específico). Es el caso, por ejemplo, de la concepción modular de Fodor o del conexionismo de McCleland y Rumelhart. En contextos teóricos similares los desfases horizontales son la regla, o bien no tiene sentido el plantear el problema en estos términos. La psicología ha entrado en una fase de micro-análisis de las operaciones que intervienen en la resolución de 
toda conducta, y esta situación impide el plantear el problema en términos de desfase horizontal.

Lo que parece claro actualmente es que no se puede hablar de modo general de desfases horizontales, ya que el concepto está demasiado marcado por los presupuestos teóricos de Piaget. Sería necesario introducir distinciones suplementarias relativas, en particular, a lo que llamamos ámbito y a los diferentes tratamientos que necesita una situación dada. Desde la perspectiva teórica que yo defiendo, y dado que la construcción de representaciones está vinculada a la competencia estructural general que aparece a ciertas edades y a las experiencias que el niño posee con respecto a determinadas realidades, los desfases son la regla. Sin embargo, ciertas conductas no pueden aparecer hasta una determinada edad, en la que se realizan transformaciones internas específicas.

P.: Piaget rechazó la existencia de conservaciones tempranas propuestas por otros autores (Mebler y Bewer, Bryant, etc.), argumentando que se trataba de las primeras formas de identidad cualitativa, pero no de conservaciones operatorias. Sin embargo, tú sostienes que el niño construye durante el periodo sensorimotor conservaciones en sentido estricto. ¿A qué se debe esta discrepancia?

R.: Pienso que se trata de una de las reacciones más defensivas de Piaget. $\mathrm{Su}$ origen reside en el temor al preformismo y su deseo implacable de explicar el desarrollo por la emergencia de estructuras nuevas. Y sin embargo, no hay estructuras sin invariantes, $¡$ tal como lo ha afirmado el mismo Piaget!

P.: Siguiendo con las diferencias, Piaget entiende el desarrollo cognitivo como una construcción progresista de esquemas y estructuras, mientas que para ti se trataría más bien de la construcción de representaciones, en el sentido de modelos o memorias internas. ¿Qué ventajas teóricas tendría tu posición y cómo determinarias la existencia de los diferentes estadios?

R.: Creo que he respondido en gran parte a esta pregunta. La noción de esquema y de estructura ha sido definida por Piaget en un nivel estructural muy abstracto, insistiendo siempre en la independencia de estos esquemas y estructuras con respecto a los objetos que sirven de contenido variable. En cambio, el concepto de representación resalta la estructuración de los contenidos.

Por otra parte, la existencia de estadios es un tema complejo, difícil de justificar. Parece que el niño llega a dominar en determinadas etapas ciertas clases de problemas, a los que ha sido sensible (o a los que puede acceder mediante sus instrumentos cognitivos), como, por ejemplo, el niño de 3,6 a 4 años en el ámbito de las seriaciones mediante encaje, o el niño de 10 años en las seriaciones «a lo Piaget». Y ocurre lo mismo con respecto a otras muchas conductas. El problema se plantea entonces en torno a si se trata de estadios generales o de estadios específicos. Yo creo, por mi parte, que los estadios son generales y caracterizan el conjunto de las conductas experimentadas por los niños.

$P .: ~ ¿ E n$ qué sentido habria que entender tu descripción del desarrollo como una secuencia de revoluciones?

R.: El término ha sido utilizado por Piaget para caracterizar el paso del período sensorimotor a la representación. Yo he empleado ese término para caracterizar las dos grandes etapas o fases que definen, a mi juicio, cada es- 
tadio (o cada adquisición de una conducta nueva), si se analiza desee sus formas elementales y fragmentarias. El término "revolución», que introduje en 1975 en el Congreso de St.-Paul de Vence sobre las «regresiones» (cf. Bever, 1982), pretendía oponerse a la concepción del desarrollo como algo continuo, "lineal", acumulativo. Se trataba de afirmar los cambios profundos que sufren los modos de tratamiento, de análisis, no sólo entre los grandes estadios, sino sobre todo en el interior del mismo estadio.

P.: Hablas en tus escritos de un empobrecimiento de las capacidades del niño, en el sentido de que el neonato presentaría una competencia cognitiva superior a la del niño de los primeros meses. Yo be encontrado un fenómeno parcialmente similar en el ámbito de la clasificación hacia los seis años y medio, apareciendo un cierto retroceso en cuanto a los criterios empleados por los niños, que volvian a los espacio-figurales (globalizantes), en vez de continuar la tendencia marcada por los sujetos de 5,6 años $y$, sobre todo, por los de 7,6 años, que usaban criterios dimensionales (forma, color, etc.). Entonces sugeri la existencia de un cambio en el modo de clasificar, influido probablemente por factores educativos o escolares. Pero volviendo a la idea de empobrecimiento, que costaría aceptar desde una concepción uniforme y progresiva del desarrollo, ¿no podrian atribuirse estos datos a deficiencias en el diseño experimental (instrumentos, variable dependiente, etc.), o existen razones teóricas que los justifiquen?

R.: Es evidente que la idea de empobrecimiento parece contradictoria con la de desarrollo. Sin embargo, así conciben ciertos biólogos, como Changeux por ejemplo, el desarrollo del sistema nervioso con su hipótesis de la "estabilidad selectiva", de modo que ciertas combinaciones sinápticas serían estabilizadas, mientras que otras serían eliminadas por la experiencia.

Los datos experimentales que evidencian un tal empobrecimiento de las capacidades cognitivas son aún poco numerosos. Y el problema es delicado, ya que supone el establecimiento de un sistema de valores que permita la comparación de diferentes modos de funcionamiento cognitivo. Y hasta que los modelos implícitos o explícitos de los investigadores no consideren la posibilidad de tales fenómenos en el proceso del desarrollo normal, pocos resultados apuntarán en esta dirección. Uno de los mejores ejemplos de empobrecimiento es la pérdida de las capacidades de discriminación fonémicos que no pertenecen a su lengua materna. Pero, ¿se trata realmente de una pérdida, dado que en determinadas condiciones el niño o el adulto parece capaz de manifestar siempre estas capacidades discriminativas? ¿Hay realmente pérdida de la capacidad discriminativa, o más bien déficit atencional selectivo como piensan actualmente ciertos autores (cf. en concreto: Jusczyk, 1985)?

Toda racionalización supone empobrecimiento (se habla también frecuentemente de un proceso de idealización) y la actividad de clasificar constituye un buen ejemplo. Para clasificar objetos hay que seleccionar ciertas dimensiones como pertinentes, e ignorar otras consideradas como irrelevantes; y lo mismo ocurre con respecto a la invarianza de ciertas propiedades, ya que hay que desdeñar ciertas dimensiones o variaciones consideradas como accidentales, para centrarse en otras tenidas por esenciales, para retomar los términos usados por Piaget. El ejemplo que tú propones es muy interesante, ya que marca la transición, el paso de la primera fase (o 
revolución) a la segunda fase del estadio de elaboración de las representaciones conceptuales. (Las distinciones que hace el niño a los 5,6 años son principalmente de naturaleza pragmática o semántica, de modo que cada objeto se caracteriza por ciertas propiedades y se yuxtapone con otros objetos con los que guarda ciertas relaciones imprecisas. En cambio, a los 6,6 años el niño inicia distinciones más abstractas: los objetos son claramente diferentes, compartiendo ciertas dimensiones comunes con otros objetos.)

Se trata del paso de una organización predominantemente semántica a una organización prioritariamente morfológica, o del paso del preconcepto al concepto. Ahora bien, ¿cómo es posible que un tal cambio de capacidades cognitivas no venga acompañado de pérdidas y de ganancia? Generalmente, los investigadores persiguen sobre todo las «ganancias» $\mathrm{y}$ las ponen de relieve, pero también se dan pérdidas como lo muestra tu experimento. Es cierto que con otro plan experimental y (o) con otras variables podrían evidenciarse otros aspectos. En todas las investigaciones que he realizado sobre la planificación y el control del gesto, he observado que ciertos aspectos evolucionan de manera monótona, mientras que otros presentan evoluciones más caóticas, tal como las célebres curvas en «U». Habría que llegar a hacer progresivamente hipótesis más específicas sobre lo que puede enriquecerse o empobrecerse y en función de qué.

P.: Aunque en España se van conociendo cada vez más tus escritos, es probable que hagamos un favor a aquellos que se inician en la lectura de tus publicaciones, recomendando alguno o algunos de tus trabajos que recojan de modo más exbaustivo tus ideas. ¿Podrias hacerlo?

R.: Sean las obras más recientes para los impacientes en comprender, sea la sucesión de algunos textos (siete u ocho) de síntesis teórica, que han jalonado mi carrera cronológicamente, para comprender una vez más una génesis (Mounoud, 1971, 1976, 1979, 1983, 1985, 1986, 1987, 1988; Mounoud y Hauert, 1982; Mounoud y Vinter, 1981).

P.: Cambiando de tema, en España se están preparando los nuevos planes de estudios $y$, en verdad, que no está resultando nada fácil su elaboración. ¿Cuál es en sus grandes lineas el plan de estudios actual de la Sección de Psicologia (objetivos generales, cursos, ciclos, etc.)? ¿Habría que hacer alguna modificación?

R.: El plan de estudios actual es sencillo. Está estructurado en tres ciclos. El primero, de dos años, es propedéutico y conduce a la obtención de la demi-licenciatura. El segundo ciclo dura igualmente dos años y concluye con la licenciatura. Finalmente, durante un quinto año puede obtenerse un diploma, realizando una investigación empírica importante y asistiendo a tres seminarios.

El plan es bastante satisfactorio desde el punto de vista de la formación académica. En cambio, lo es menos desde el punto de vista de la formación práctica. Es cierto que hay trabajos prácticos durante el primer y segundo ciclo; pero sería perentorio introducir cursillos durante el segundo ciclo, y sobre todo dos o tres años de trabajo como psicólogos ayudantes en instituciones vinculadas con la Universidad después de la licenciatura. Yo mismo di algunos pasos en esta dirección durante los dos años que fui presidente de la Sección de Psicología y actualmente existen transacciones en este sentido. 
P.: Y como otros imperativos (limitación de espacio, por ejemplo) y no el agotamiento de temas, nos obligan a concluir esta entrevista, permiteme agradecerte tu disponibilidad y plantearte la última pregunta un tanto tópica, pero, a mi juicio, indispensable: ¿ Hacia dónde va la Psicología cientifica?

R.: ¡Un problema más de desarrollo! Como todas las disciplinas, la psicología sufre múltiples influencias. La sociedad actual tiene que hacer frente a numerosos problemas, y en particular el envejecimiento (gerontología), y la psicología debe contribuir en el estudio de este gran problema. El cambio ya se ha producido, estudiándose el desarrollo a lo largo de toda la vida (life span perspective). Pero hay más cosas que hacer, y es evidente que este sector de la psicología va a tener un crecimiento considerable. Desde los años sesenta, Ajuriaguerra y Tissot se interesaron en Ginebra en ciertos problemas de envejecimiento; y actualmente el equipo de trabajo del Dr. Richard, profesor de nuestra Facultad, realiza numerosos trabajos en este ámbito. Este es precisamente el tema de la próxima Conferencia de la Asociación de Psicología Científica de Lengua Francesa, que tendrá lugar en Ginebra en 1989.

Otra influencia capital que incide actualmente en la psicología es la de las neurociencias. Es este sentido hay que resaltar el rápido desarrollo que la neuropsicología experimental ha conocido estos últimos años, especialmente en Canadá, Inglaterra e Italia. Pienso que se trata de un campo muy prometedor en el que nada hay hecho desde el punto de vista de la psicología evolutiva. Uno de mis colaboradores, Olivier Koenig, acaba de terminar su tesis de doctorado sobre el efecto Stroop en niños de 6 a 15 años con presentación lateralizada hemicámpica.

Y hay también las repercusiones del progreso tecnológico, que han provocado siempre progresos fantásticos en el estudio del comportamiento. La posibilidad de registrar tridimensionalmente los movimientos, por ejemplo, está hoy en plena expansión, y gracias a estos medios se hace accesible el estudio de nuevos problemas. No quisiera terminar con una nota pesimista, pero a veces estoy desconcertado por la proliferación de investigaciones, de congresos y de revistas frecuentemente muy especializadas, y la dificultad de llevar a cabo en un tal torbellino tentativas de sintesis, que resultan totalmente necesarias y que dan un poco de persepectiva, de relieve a este campo cautivador que será siempre el desarrollo psicológico del niño.

Para terminar, quisiera dar las gracias aquí a los numerosos colaboradores, colegas y estudiantes que me han ayudado y estimulado a lo largo de mi carrera científica, y sin los cuales no hubiera llegado a realizar mis trabajos. Deseo mencionar en particular a dos colaboradores principales que trabajan conmigo desde hace muchos años: Claude-Alain Hauert y Annie Vinter, a los que agradezco encarecidamente su notable contribución, su apoyo y su fiel amistad. Quisiera mencionar también a mi colega y amigo Paolo Viviani, con el que trabajo desde hace más de quince años y me siento igualmente en deuda. 


\section{Referencias}

BEVER, T. G. (comp.) (1982). Regressions in mental development: basic phenomena and theories. Hillsdale, N. J.: Erlbaum.

ChangeuX, J. P. (1983). L'bome neuronal. París: Fayard.

FODOR, J. A. (1983). The modularity of mind. Cambridge, M. A.: MIT Press.

JUSZCYK, P. W. (1985). On characterizing the development of speech perception. En J. Mehler y R. Fox (comps.): Neonate cognition: beyond the blooming buzzing confusion. Hillsdale, N. J.: Erlbaum.

[1] Mounoud, P. (1971). Développement des systémes de représentation et de traitement chez l'enfant. Bulletin de Psychologie, 25 (5-7), 261-277.

[2] MounOud, P. (1976). Les révolutions psychologiques de l'enfant. Archives de Psychologie, 44, $103-114$.

[3] MOUNOUd, P (1979). Développement cognitif: construction de structures nouvelles ou construction d'organisations internes. Bulletin de Psychologie, 33, 107-118.

MOUNOUD, P. (1983). L'evolution des conduites de préhension comme illustration d'un modéle de développement. En S. de Schonen: Le développement dans la premiére année. Paris: Presses Universitaires de France.

Mounoud, P. (1985). Similarities between developmental sequences at different age periods. En I. Levin (Comp.): Stage and structure. Norwood: Ablex.

Mounoud, P. (1986). Action and cognition. Cognitive and motor skills in a developmental perspective. En M. G. Wade y H. T. A. Whiting (comps.): Motor development in children. Dordrecht: Nijhoff.

Mounoud, P. (1987). L'utilisation du milieu et du corps propre par le bébé. En J. Piaget, P. Mounoud y J. P. Bronkart (comps.): La psychologie. París: Encyclopédie de la Pléiade, Gallimard.

MOUNOUD, P. (1988). The ontogenesis of different types of thoughts. Languaje and motor behavior as non-especific manifestations. En I. L. Weiskrantz (comp.): Thought without language. Oxford: Oxford University Press.

MOUNOUD, P. y HAUERT, C. A. (1982). Development of sensori-motor organization in young children: grasping and lifting objects. En G. Forman (comp.): Action and thought: form sensori-motor schemes to symbolic operations, Nueva York: Academic Press.

MOUNOUD, P. y VINTER, A. (1981a). Representation and sensori-motor development. En G. Butterworth (comp.): Infancy and epistemology: an evaluation of Piaget's theory. Brighton: The Harvester Press.

[1] Artículo traducido al inglés en B. Inhelder y H. Chipman (comps.) (1976): Piaget reader. Nueva York: Springer Velag.

[2] Artículo traducido al inglés en T. G. Bever (1982): Regressions in mental development: basic phenomena and theories. Nueva York: Erlbaum.

[3] Artículo traducido al inglés en I. E. Siegel, D. M. Brodzinsky y R. M. Golinkiff (comps.) (1981). New directions in piagetian theory and practice. Hillsdale, N. J.: Erlbaum. 